\title{
Construction of CNA35 Collagen-Targeted Phase- Changeable Nanoagents for Low-Intensity Focused Ultrasound-Triggered Ultrasound Molecular Imaging of Myocardial Fibrosis in Rabbits
}

Citation for published version (APA):

Zhou, Q., Zeng, Y., Xiong, Q., Zhong, S., Li, P., Ran, H., Yin, Y., Reutelingsperger, C., Prinze, F. W., \& Ling, Z. (2019). Construction of CNA35 Collagen-Targeted Phase-Changeable Nanoagents for LowIntensity Focused Ultrasound-Triggered Ultrasound Molecular Imaging of Myocardial Fibrosis in Rabbits. ACS Applied Materials \& Interfaces, 11(26), 23006-23017. https://doi.org/10.1021/acsami.9b05999

Document status and date:

Published: 03/07/2019

DOI:

10.1021/acsami.9b05999

Document Version:

Publisher's PDF, also known as Version of record

\section{Document license:}

Taverne

\section{Please check the document version of this publication:}

- A submitted manuscript is the version of the article upon submission and before peer-review. There can be important differences between the submitted version and the official published version of record. People interested in the research are advised to contact the author for the final version of the publication, or visit the $\mathrm{DOI}$ to the publisher's website.

- The final author version and the galley proof are versions of the publication after peer review.

- The final published version features the final layout of the paper including the volume, issue and page numbers.

Link to publication

\footnotetext{
General rights rights.

- You may freely distribute the URL identifying the publication in the public portal. please follow below link for the End User Agreement:

www.umlib.nl/taverne-license

Take down policy

If you believe that this document breaches copyright please contact us at:

repository@maastrichtuniversity.nl

providing details and we will investigate your claim.
}

Copyright and moral rights for the publications made accessible in the public portal are retained by the authors and/or other copyright owners and it is a condition of accessing publications that users recognise and abide by the legal requirements associated with these

- Users may download and print one copy of any publication from the public portal for the purpose of private study or research.

- You may not further distribute the material or use it for any profit-making activity or commercial gain

If the publication is distributed under the terms of Article $25 \mathrm{fa}$ of the Dutch Copyright Act, indicated by the "Taverne" license above,

Download date: 26 Apr. 2023 


\title{
Construction of CNA35 Collagen-Targeted Phase-Changeable Nanoagents for Low-Intensity Focused Ultrasound-Triggered Ultrasound Molecular Imaging of Myocardial Fibrosis in Rabbits
}

\author{
Qin Zhou, ${ }^{\dagger}$ Yalin Zeng, ${ }^{\dagger}$ Qingsong Xiong, ${ }^{\dagger}$ Shigen Zhong, ${ }^{\ddagger}$ Pan Li, ${ }^{\ddagger}$ Haitao Ran, ${ }^{\ddagger}$ Yuehui Yin, ${ }^{\dagger}$ \\ Chris Reutelingsperger, ${ }^{\S}$ Frits W. Prinze, ${ }^{\Uparrow}$ and Zhiyu Ling*, ${ }^{*}, \neq 0$ \\ ${ }^{\dagger}$ Department of Cardiology, Second Affiliated Hospital of Chongqing Medical University, Chongqing 400010, P. R. China \\ ${ }^{*}$ Institute of Ultrasound Imaging, Chongqing Key Laboratory of Ultrasound Molecular Imaging, The Second Affiliated Hospital of \\ Chongqing Medical University, Chongqing 400010, P. R. China \\ ${ }^{\S}$ Department of Physiology, Cardiovascular Research Institute Maastricht, Maastricht University, P.O. Box 616, 6200 MD, \\ Maastricht, The Netherlands \\ "Department of Biochemistry, Cardiovascular Research Institute Maastricht, University of Maastricht, P.O. Box 616, 6200 MD, \\ Maastricht, The Netherlands
}

\section{Supporting Information}

\begin{abstract}
Myocardial fibrosis plays an important role in the development of heart failure and malignant arrhythmia, which potentially increases the incidence of sudden cardiac death. Therefore, early detection of myocardial fibrosis is of great significance for evaluating the prognosis of patients and formulating appropriate treatment strategies. Late gadoliniumenhanced magnetic resonance imaging is considered as the currently effective strategy for noninvasive detection of myocardial fibrosis, but it still suffers from some critical issues. In this work, multifunctional CNA35-labeled perfluoropentane nanoparticles (CNA35-PFP NPs) have been elaborately designed and constructed for molecular imaging

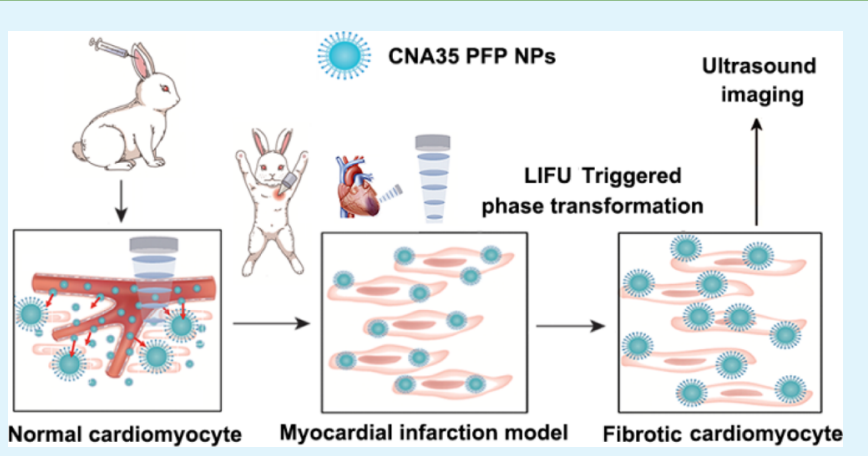
of fibrotic myocardium based on ultrasound imaging. These as-constructed CNA35-PFP NPs are intravenously infused into rabbit circulation with an animal model of myocardial infarction. Especially, these targeted CNA35-PFP NPs with nanoscale size could efficiently pass through the endothelial cell gap and adhere to the surface of fibroblasts in the fibrotic myocardium. Importantly, followed by low-intensity focused ultrasound irradiation on the myocardium, these intriguing CNA35-PFP NPs could transform from liquid into gaseous microbubbles, which further significantly enhanced the ultrasound contrast in the fibrotic area, facilitating the detection by diagnostic ultrasound imaging. Therefore, this work provides a desirable noninvasive, economical, and real-time imaging technique for the assessment of cardiac fibrosis with diagnostic ultrasound based on the rational design of liquid-to-gas phase-changeable nanoplatforms.
\end{abstract}

KEYWORDS: myocardial fibrosis, CNA35, fluorocarbon nanoparticles, ultrasound molecular imaging, diagnosis, nanomedicine

\section{INTRODUCTION}

Myocardial fibrosis refers to the pathological process in which collagen fibers are excessively accumulated in the myocardium to alter the structure of normal myocardial tissue. ${ }^{1}$ Myocardial fibrosis is involved in various diseases such as myocardial ischemia, cardiomyopathy, and myocarditis. ${ }^{2,3}$ The solid evidences have demonstrated that myocardial fibrosis can lead to serious diseases such as heart failure, malignant arrhythmia, and sudden cardiac death. ${ }^{4-6}$ Therefore, it is highly important to confirm the degree and location of myocardial fibrosis in evaluating the prognosis of patients and developing treatment options. Currently, the golden standard for diagnosing myocardial fibrosis is myocardial biopsy, but this invasive procedure is cumbersome and needs a long recovery time. Especially, the extent and location of the fibrosis cannot be clearly determined by this approach. Another approach for noninvasive detection of myocardial fibrosis is based on the late gadolinium-enhanced magnetic resonance imaging (MRI), ${ }^{7}$ which can show the location and extent of myocardial fibrosis. However, MRI has a poor spatial resolution and is also susceptible to metallic implants. Moreover, MRI is contraindicated in patients with renal insufficiency and claustrophobia. ${ }^{8}$ Therefore, it is still highly urgent and necessary for developing an efficient and non-

Received: April 7, 2019

Accepted: May 28, 2019

Published: May 28, 2019 
invasive method for qualitative and quantitative diagnosis of myocardial fibrosis.

As a new emerging imaging technique, ultrasound molecular imaging combines the unique intrinsic advantages of ultrasound imaging with the capability of molecular imaging. ${ }^{9,10}$ The principle is based on the specific molecular markers that can be attached to the surface of nanosized contrast agents of ultrasound, so these nano-contrast agents are capable of targeting the specific antigen or antibody of the disease-related cells or tissues. ${ }^{11-13}$ After the nano-contrast agents accumulate to the specific location by intravenous injection, low-intensity focused ultrasound (LIFU) is used to induce the nano-contrast agents undergoing liquid-gas phase change and converting into microbubbles, which can be easily detected by diagnostic ultrasound. ${ }^{14-16}$ This diagnostic-imaging approach provides a novel noninvasive, economical, real-time imaging technique for inspecting the specific molecules in the disease tissue. ${ }^{17-19}$ Recently, several molecular probes have been designed for ultrasound molecular imaging in animal models with liver tumors, retinoblastoma and liver fibrosis, but the rational design of phase-changeable nano-contrast agents on the ultrasound molecular imaging of myocardial fibrosis is still highly challenging. ${ }^{20-24}$

Type I collagen is the main collagen component in fibrotic myocardial tissue, accounting for $80-85 \%$ of the total collagen content. ${ }^{25-27}$ Extensive research studies have demonstrated that type I collagen is excessively expressed in myocardial fibrosis caused by various diseases. ${ }^{28-30}$ Detection of type I collagen can be used to assess the content of myocardial fibrosis. Recently, a fluorescent probe CNA35 $5^{31-33}$ has been used for type I collagen labeling. It is a $35 \mathrm{kDa}$ protein extracted from the surface of Staphylococcus aureus, which specifically bind to type I collagen. Especially, CNA35 has been further developed as a molecular probe to fabricate a targeted contrast agent of MRI and computed tomography (CT) for the imaging of myocardial fibrosis. ${ }^{34,35}$ For instance, Chris Reutelingsperger et al. ${ }^{36}$ developed ${ }^{99 \mathrm{~m}} \mathrm{Tc}-(\mathrm{CO})_{3}$ His-CNA35 to localize tumor vascular collagen for molecular imaging of tumor vasculature. On the basis of the fast development of ultrasound molecular imaging and type I collagen-based targeting design, it was hypothesized that liquid-gas phasechangeable fluorocarbon nanoparticles carrying type I collagenbinding CNA35 could pass through the myocardial endothelium and then bind to the fibrotic myocardium (Figure 1). After the liquid-gas phase change as induced by LIFU irradiation, these fluorocarbon nanoparticles could be converted into microbubbles, which could be detected by diagnostic ultrasound, achieving the goal of real-time molecular imaging of cardiac fibrosis with ultrasound. Therefore, the specific goal of this work is to develop CNA35-labeled perfluoropentane nanoparticles (designated as CNA35-PFP NPs) and evaluate their specific functionality of selective, noninvasive, and real-time imaging of cardiac fibrosis with diagnostic ultrasound.

\section{MATERIALS AND METHODS}

2.1. Fabrication of Fluorocarbon Nanoparticles (PFP NPs) and CNA35-Labeled Perfluoropentane Nanoparticles (CNA35PFP NPs). 1,2-Dipalmitoyl-sn-glycero-3-phosphocholine (DPPC, 10 mg; Avanti Polar Lipids, Alabaster, AL, USA), $4 \mathrm{mg}$ of 1,2dioctadecanoyl-sn-glycero-3-phosphoethanolamine (DSPE; Avanti Polar Lipids, Alabaster, AL, USA), 3 mg of 1,2-distearoyl-sn-glycero3-phosphorylglycerol (DSPG; Avanti Polar Lipids, Alabaster, AL, USA), and $3 \mathrm{mg}$ of cholesterol (Sigma, St. Louis, MO, USA) were

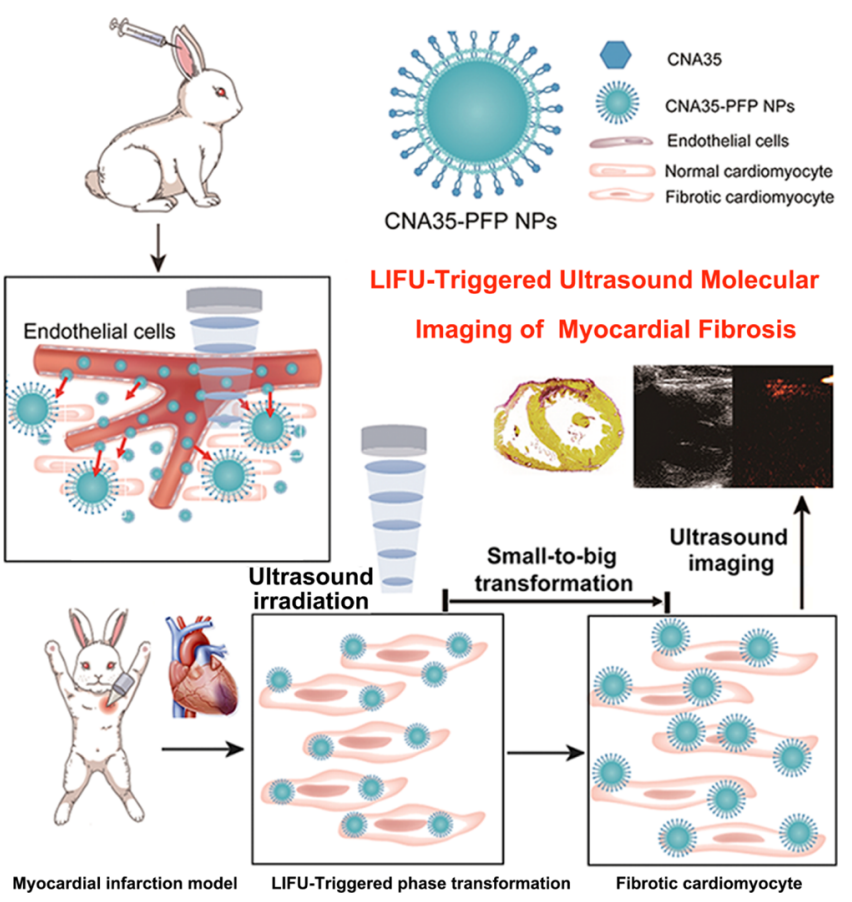

Figure 1. Schematic illustration of the design of CNA35-PFP NPs combined with LIFU to detect myocardial fibrosis. Nanoscale CNA35-PFP NPs could pass through the myocardial vascular endothelium and target the fibrotic myocardium. Under the external LIFU irradiation, CNA35-PFP NPs could phase-transform into microbubbles and enhance ultrasound molecular imaging of myocardial fibrosis.

dissolved with $10 \mathrm{~mL}$ of trichloromethane (Chongqing Chuandong Chemicals, Chongqing, China), and then the organic solvent was removed with a rotary vacuum evaporator (RE-52A, Yarong, Shanghai, China). After adding $200 \mu \mathrm{L}$ of liquid perfluoropentane (PFP; Elf Atochem, France) to the liquid membrane solution, fluorocarbon lipid nanoparticles were prepared by pulse vibration for 6 min with a sonicator (VCY-500, Shanghai Yanyong Ultrasonic Instrument Co., Ltd., Shanghai, China). Finally, nanosized and uniform contrast agents were obtained by centrifugation in a refrigerated centrifuge (5804R; Eppendorf, Hamburg, Germany) at $4{ }^{\circ} \mathrm{C}$ (6000 rpm, $5 \mathrm{~min}$ three times). After each time of centrifugation, the perfluoropentane nanoparticles (PFP NPs) were washed and collected with $4 \mathrm{~mL}$ phosphate-buffered saline (PBS) for further study.

To fabricate CNA35-labeled perfluoropentane nanoparticles (CNA35-PFP NPs), the typical carbodiimide method was used. DSPE-PEG2000- $\mathrm{NH}_{2}$ (4 mg, Xi'an Ruixi Biotechnology Co., Ltd. Xian, China) was used to replace DSPE during the process of nanoparticle synthesis, which was mixed with a small amount of DiI (Beyotime Biotechnology, Shanghai, China). The other steps were the same as described above. CNA35-FITC $(200 \mu \mathrm{L}, 1.85 \mathrm{mg} / \mathrm{mL}$, kindly provided by C.R., Department of Biochemistry, University Maastricht, The Netherlands) was mixed with $2 \mathrm{~mL}$ of as-prepared phase-changeable nanoparticles. The suspension was incubated in an ice bath for $24 \mathrm{~h}$, and then concentrated by centrifugation and washed with $4 \mathrm{~mL}$ PBS for three times. This whole process was conducted in the dark to avoid the loss of fluorescein isothiocyanate (FITC) fluorescence activity.

2.2. Characterization of PFP NPs. The diameter and distribution of the nano-contrast agents were observed with an optical microscope (Olympus, Tokyo, Japan) and a confocal laser scanning microscope (A1R-Si; Nikon, Tokyo, Japan). The morphology and microstructure of the nanoparticles were observed by transmission electron microscopy (TEM) (H-7500; Hitachi, Tokyo, Japan).The average diameter and zeta potential of the nanoparticles 
were measured by a dynamic light scattering analyzer (Malvern Instruments, Malvern, UK). Flow cytometry was used to assess the connectivity of CNA35 and PFP NPs.

2.3. Phase Transition and Ultrasound Imaging of CNA35PFP NPs. To investigate the phase-transition behavior of CNA35PFP NPs, we performed both heating and focused ultrasound irradiation to induce the phase transition. The CNA35-PFP NPs were heated at $37,40,45$, and $50{ }^{\circ} \mathrm{C}$ for 1,5 , and 10 min using a constant temperature water bath heater. Esaote Mylab90 (Genoa, Italy) ultrasonic diagnostic system (probe LA523, frequency 5-9 MHz) was used to compare the gray-scale changes between the B-mode and contrast-enhanced ultrasound (CEUS) group. The phase-transition process of the nano-contrast agents was observed under a microscope.

In addition, the phase transition of CNA35-PFP NPs was induced in vitro by low-intensity focused ultrasound (LIFU; LMSC051 ACA; Institute of Ultrasound Imaging, Chongqing Medical University, Chongqing, China). First, these CNA35-PFP NPs were diluted and added into a perforated agar gel phantom (3\% agar content), and the surface of the LIFU probe was coated with an ultrasonic coupling agent and the focus of the LIFU was positioned at the center of the nano-contrast agents. Then, the sample was irradiated by LIFU for 1$4 \mathrm{~min}$ at the power of $1-4 \mathrm{~W} / \mathrm{cm}^{2}$. The ultrasound images before and after each LIFU irradiation were recorded for further analysis.

2.4. Animal Model Establishment. New Zealand white rabbits (provided by the Animal Center of Chongqing Medical University) were chosen to establish an in vivo myocardial infarction model. All experimental procedures were conducted in accordance with local guidelines on the ethical use of animals and the National Institutes of Health "Guide for the Care and Use of Laboratory Animals" (NIH publication no. 80-23, revised 1996). The animal experiments were approved by the Animal Experimental Committee of Chongqing Medical University. Female New Zealand white rabbits (average weight of $2 \mathrm{~kg}$ ) were randomly selected and anesthetized by intravenous injection of $3 \%$ pentobarbital sodium $(1 \mathrm{~mL} / \mathrm{kg})$. Lidocaine $(2 \mathrm{mg} / \mathrm{kg})$ was injected via the ear vein for preventing malignant arrhythmias before surgery. A median sternotomy was performed to expose the heart. Myocardial infarction was induced by ligation of the middle segment of the left anterior descending coronary artery with a 5-0 silk suture. ST-segment elevation of the precordial leads in the ECG was considered successful. One month after operation, myocardial tissue of the infarct-related area was collected for pathological examination.

2.5. Ex Vivo Localization, Imaging, and Quantitative Analysis of CNA35-FITC. Frozen sections $(10 \mu \mathrm{m})$ of an infarcted myocardium and a normal myocardium ( $n=5$ for each group) were used to compare the collagen expression. The nucleus and collagen fibers were localized using 4',6-diamidino-2-phenylindole (DAPI) and CNA35-FITC, respectively. CNA35-FITC $(10 \mu \mathrm{L})$ was added to the sections, which was further incubated for $15 \mathrm{~min}$ at room temperature and then washed with PBS three times to remove the unbound CNA35-FITC. An antifluorescent quencher was added to cover the sections with a coverslip for sealing. The stained sections were observed with a fluorescence confocal microscope. Picrosirius red (PSR) was used to stain the same sections after CNA35-FITC imaging and the results were compared with the fluorescent images.

The corresponding myocardial fraction was manually selected under the microscope field for analysis. The percentage of collagen in each field was calculated using ImageJ software (1.48 V, NIH, Bethesda, MD, USA). All measurements were performed five times per image and the average of the results was calculated.

2.6. In Vitro and in Vivo Safety Evaluation of CNA35-PFP NPs. To investigate the safety of CNA35-PFP NPs, an in vitro cytotoxicity test and an in vivo safety evaluation on healthy rabbits were performed. For in vitro safety evaluation, rat primary cardiac fibroblasts (CFs) were isolated from neonatal Sprague Dawley rats as we described previously. ${ }^{37}$ CCK- 8 assay was used to examine the cell viability of CFs after incubation with various concentrations of CNA35-PFP NPs $(0.6,1.25,2.5$, and $5 \mathrm{mg} / \mathrm{mL})$ for $24 \mathrm{~h}$. For the in vivo safety test, $4 \mathrm{~mL}$ of CNA35-PFP NPs were injected from the ear vein into five normal rabbits. Forty-eight hours after injection, the rabbits were sacrificed and the heart, lung, liver, kidney, and spleen were collected for histological evaluation.

2.7. In Vivo Detection of Type I Collagen Fibers Targeted by CNA35-FITC-PFP NPs-Dil. Ten rabbits with myocardial infarction were randomly divided into two groups ( $n=5$ for each group) 4 weeks after induction of myocardial infarction. In group one, $4 \mathrm{~mL}$ of diluted PFP NPs-DiI was injected through the ear vein. In the other group, $4 \mathrm{~mL}$ of FITC-labeled CNA35 nanoparticles (CNA35-FITCPFP NPs-DiI) were also injected by intravenous injection. Ten normal rabbits used as controls were also randomly divided into two groups and received PFP NPs-DiI or CNA35-FITC-PFP NPs-DiI injection, respectively. One hour after injection, the hearts were harvested in the dark, and the left ventricle frozen sections were prepared. The sections were stained with DAPI and analyzed by fluorescence confocal microscopy to localize targeted CNA35-FITCPFP NPs-Dil.

2.8. In Vivo Phase Transition of CNA35-PFP NPs and Ultrasound Molecular Imaging of Myocardial Fibrosis. Ten more rabbits with myocardial infarction were subject to ultrasound molecular imaging procedures. Echocardiography was performed with a commercially available echocardiographic system (Esaote MyLab 90, Genoa, Italy) equipped with a LA523 probe. The chamber size, wall thickness, amplitude of wall motion, and the echo intensity of anterior wall were recorded. The location of the infarcted area was observed according to the wall motion and wall thickness. Ten rabbits were randomly divided into two groups; PFP NPs or CNA35-PFP NPs were injected through the ear vein, respectively. Five minutes after injection, the rabbits in both groups received LIFU irradiation directed to the infarcted area for $3 \mathrm{~min}$. Ultrasound images at baseline, 5 min after the nanoparticles injection, and after LIFU irradiation were recorded and compared.

2.9. Statistical Analysis. The imaging signal of the corresponding regions in B-mode and contrast mode was calculated with an ultrasound imaging analyzer (DFY-II; Institute of Ultrasound Imaging, Chongqing, China) ${ }^{16,38}$ All data were recorded as mean \pm standard deviation. Data analysis was performed using Stata for Windows version 15.0 (Stata Corp LLC, College Station, TX, USA). Statistical images were analyzed with GraphPad Prism for Windows version 6.0 (GraphPad, Inc., San Diego, CA, USA). The $t$-test was used to compare the thickness of the infarcted myocardium and noninfarcted area. Also, Student's $t$-test was performed to determine differences of fibrosis between the control group and the experimental group. One-way ANOVA was used to test the differences of mean gray-scale values of ultrasound molecular imaging in myocardial infarction rabbits that had received CNA35-NP/CNA35-PFP NPs at baseline, 5 min after injection, and after LIFU irradiation. A two-sided probability value $p<0.05$ was considered a statistically significant difference.

\section{RESULTS AND DISCUSSION}

3.1. Characterization of PFP NPs and CNA35-PFP NPs. In this work, we successfully synthesized CNA35-labeled perfluoropentane nanoparticles (CNA35-PFP NPs) carrying a CNA35 molecular probe by a carbodiimide method. Myocardial fibrosis is a common pathological change in most myocardial diseases. ${ }^{39,40}$ A normal myocardium is gradually replaced by fibroblasts and collagen fibers of which type I collagen fibers are the main components. As a molecular probe, the ability of CNA35 to target type I collagen in the myocardium has been demonstrated in basic research and animal studies. ${ }^{36,41,42}$ The liquid fluorocarbon/phospholipid film-forming material is doped with a phospholipid carrying an amino group at the terminals. In the experimental procedure, we dissolved 1-ethyl-3-(3-dimethylaminopropyl)carbodiimide hydrochloride and $\mathrm{N}$-hydroxysuccinimide in a certain ratio in 2-( $\mathrm{N}$-morpholino)ethanesulfonic acid $(\mathrm{pH}=5.2)$ solution, and the mixture activated the carboxyl group at the end of CNA35FITC. During the co-incubation, the amino termini of the 
nanoparticles were bound to the carboxy terminus of CNA35FITC. In the preparation of nanoparticles, we labeled the nanoparticles with DiI, a red fluorescent lipid dye, allowing to determine colocalization of CNA35-FITC with the DiI-stained nanoparticles. As observed by an optical microscope, PFP NPs were evenly distributed on the slide glass, and the individual size was uniform. After the modification of FITC and DiI, the final CNA35-FITC-PFP NPs-DiI shows both green fluorescence (FITC, Figure 2a) and red fluorescence (DiI, Figure 2b)
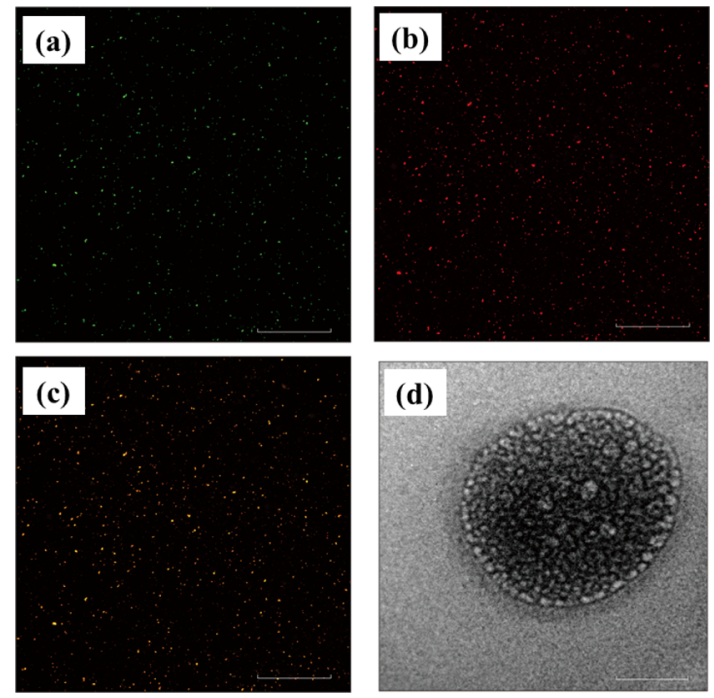

Figure 2. $(a-c)$ Fluorescent images of CNA35-FITC-PFP NPs-DiI showing different fluorescences originating from the (a) FITC component and the (b) DiI component, and (c) is the merged image of $(a, b)$ images $(100 \times)$. (d) TEM image of PFP NPs. Scale bar: $100 \mathrm{~nm}$.

under the observation of confocal laser scanning microscopy. The merged fluorescence images further demonstrate the successful construction of CNA35-FITC-PFP NPs-DiI with surface CNA35 targeting modification (Figure 2c).

Recently, with the extensive application of the CEUS technique in clinical practice, ultrasound molecular imaging and therapeutic research have received increasing attention. ${ }^{43}$ As an emerging noninvasive method, ultrasonic molecular imaging has an important clinical application prospect because of its simplicity, targeting, and real-time feature. ${ }^{44}$ At present, micron-scale ultrasound microbubbles are mainly used as tracers of blood flow in clinic practice, ${ }^{45}$ but they have some shortcomings in practical application, such as short in vivo circulation time, vulnerable to the blood flow shear stress, and difficulty in traversing the endothelium because of the dimension limitation. In this work, the average size of a single PFP nanoparticle under TEM observation was approximately $250 \mathrm{~nm}$ (Figure 2d).The average hydrodynamic particle size of PFP NPs and CNA35-PFP NPs were respectively determined to be $255.86 \pm 3.13$ and $295.62 \pm 4.19 \mathrm{~nm}$ as tested by dynamic light scattering (Figure S1a). The nanosized CNA35 nanoparticles hypothetically pass the vascular endothelium and target fibrosis in the ischemic myocardium, allowing molecular imaging of fibrotic lesions.

In addition, the mean zeta potential of PFP NPs and CNA35-PFP NPs were $-1.73 \pm 0.46$ and $1.79 \pm 0.13 \mathrm{mV}$, respectively (Figure S1b and Table S1). Flow cytometry was further used to determine the connection rate of CNA35 onto
PFP NPs, where as high as $86 \%$ connection rate was recorded to guarantee the further efficient targeting detection of type I collagen fibers (Figure 3).

3.2. In Vitro Phase Transition and Ultrasound Imaging of CNA35-PFP NPs. As shown in Figures 4 and 5 , both thermal effect and LIFU irradiation could induce liquid-gas phase transition of as-synthesized CNA35-PFP nanoagents. It has been found that the initial CNA35-PFP NPs were difficult to be distinguished under optical microscopic observation because of the small particle size. Importantly, the increased temperature triggered the phase change from liquid NPs to gaseous microbubbles as demonstrated by the presence of large microbubbles under the optical microscopic observation (Figure 4a). For ultrasound imaging, such a liquid-gas phase-changeable process significantly enhanced the echo intensity in the agar gel model filled with CNA35PFP NPs under B-mode and CEUS mode (Figure 4b), which was also based on the increased temperature that triggered the gaseous change of the encapsulated PFP component within the targeted nanoagents, demonstrating the temperature-sensitive ultrasound-imaging performance.

In this work, lipids with desirable elasticity were used as nanoparticles' shells, which could reduce the ultrasonic power required to achieve a phase transition and effectively prevent damage to the body caused by excessive irradiation power. It has been demonstrated that PFP is an idea material for microbubble construction and its boiling point of the phasechangeable PFP is $29^{\circ} \mathrm{C},{ }^{46-49}$ which can be gasified at room temperature. However, the nanoparticles' phase-changeable temperature was elevated after being encapsulated by the lipid shell. The study of Rapoport et al. ${ }^{50}$ showed that the temperature threshold of the phase transition of liquid fluorocarbon was related to the diameter of the particles. The smaller the size of the particles, the higher the temperature required for the phase transition. At normal body temperature of around $37^{\circ} \mathrm{C}$, only when the diameter reached $4 \mu \mathrm{m}$ did the phase transformation of the encapsulated PFP occur. In the heating-phase change experiment, no significant phase transition of the nanoagents was observed when the nanoparticles were heated to $37^{\circ} \mathrm{C}$ for $10 \mathrm{~min}$. We further tested the stability of the nanoparticles in vitro by heating at $37.5^{\circ} \mathrm{C}$ for 30 and $60 \mathrm{~min}$. No obvious phase change was observed even when heated at $37.5{ }^{\circ} \mathrm{C}$ for $60 \mathrm{~min}$ (Figure S2), indicating that these nanoparticles were stable in the human body.

Importantly, the introduction of LIFU irradiation could also trigger the liquid-gas phase change of CNA35-PFP NPs as demonstrated by the post-generation of large amounts of microbubbles with large particle size (Figure 5a) at the irradiation power density of $1-2 \mathrm{~W} / \mathrm{cm}^{2}$. The irradiation with LIFU at different power densities and durations also revealed that the echo intensity of CNA35-PFP NPs increased with time at the power density of $1-2 \mathrm{~W} / \mathrm{cm}^{2}$. When the power density was set as $3 \mathrm{~W} / \mathrm{cm}^{2}$, the echo intensity reached its peak after 3 min of LIFU irradiation, and then decreased at the 4th min. In the group of $4 \mathrm{~W} / \mathrm{cm}^{2}$, the time of the echo intensity reaching its peak was $2 \mathrm{~min}$, which began to decrease at the 3rd $\min$. Comparatively, the echo intensity at the $2 \mathrm{nd} \min$ in the 4 $\mathrm{W} / \mathrm{cm}^{2}$ group was lower than that in the $3 \mathrm{~W} / \mathrm{cm}^{2}$ group at the 3rd min (Figure $5 \mathrm{~b}$ ). This result demonstrates that these asfabricated CNA35-PFP NPs are featured with an LIFUsensitive phase-changeable property and corresponding ultrasound-imaging performance. Quantitative analysis demonstra- 

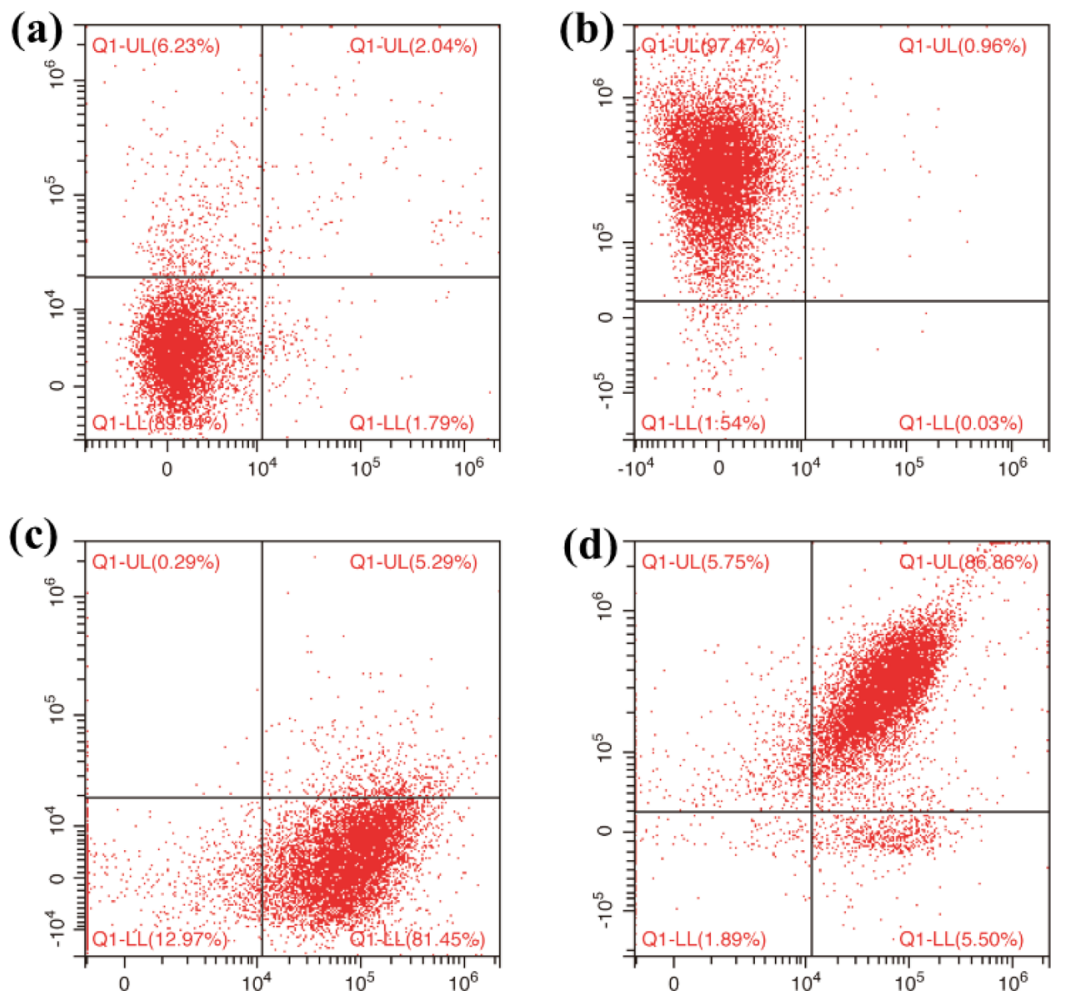

Figure 3. Analysis of CNA35-PFP NP connectivity by flow cytometry, including (a) negative control group, (b) PFP NPs-DiI group, (c) CNA35FITC-PFP NPs group, and (d) CNA35-FITC-PFP NPs-DiI group. The connection rate was determined to be $86 \%$.
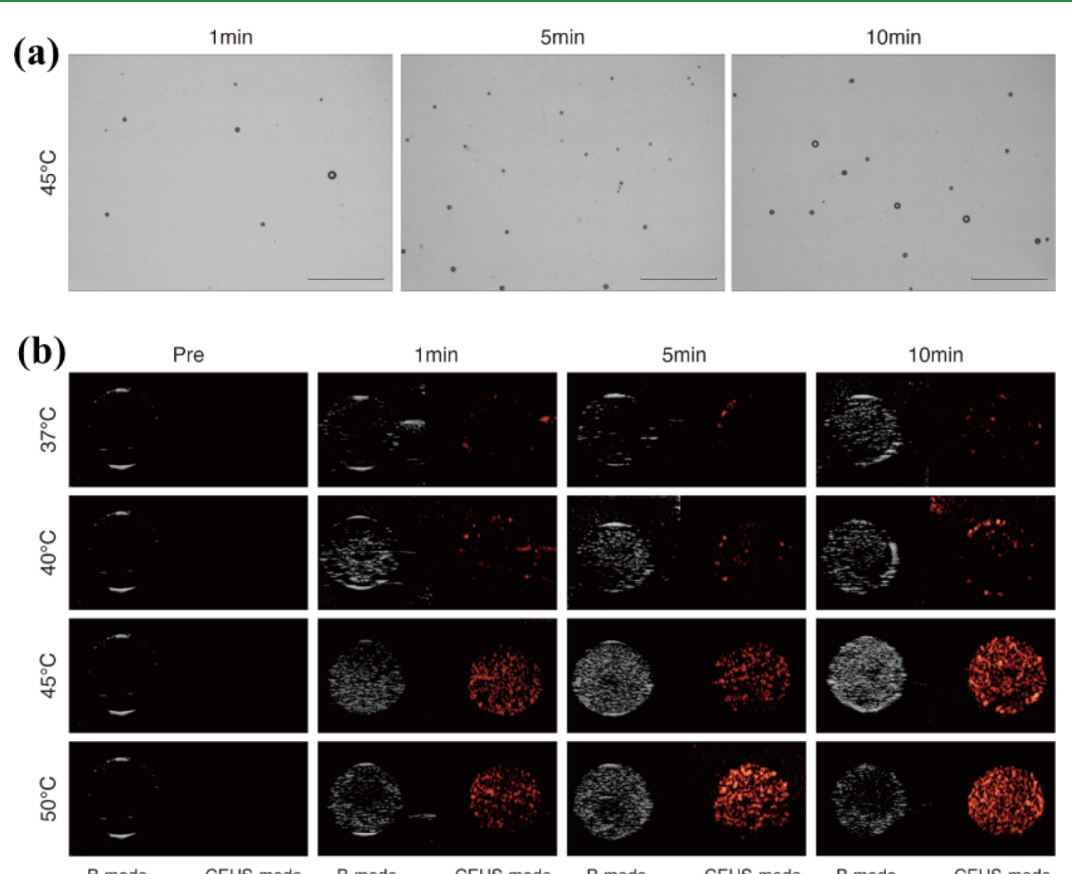

Figure 4. In vitro phase transition and ultrasound imaging of CNA35-PFP NPs. (a) Optical microscopic distribution image of CNA35-PFP NPs phase transformation by heating at $45{ }^{\circ} \mathrm{C}(100 \times)$. (b) CEUS imaging effect of each group in ultrasonic mode (B-mode and CEUS mode) after heating in a water bath.

ted that ultrasound imaging of fluorocarbon nanoparticles was more obvious when exposed to $3 \mathrm{~W} / \mathrm{cm}^{2}$ for $3 \mathrm{~min}$, indicating that most of the nanoparticles experienced a phase transition. This phenomenon is consistent with the experimental results of Zhu et al., ${ }^{51}$ which proves that these nanoparticles could undergo phase transformation under certain conditions to achieve the desirable imaging results. Therefore, we chose LIFU power density of $3 \mathrm{~W} / \mathrm{cm}^{2}$ and irradiation time of $3 \mathrm{~min}$ for in vivo assessment.

\subsection{Fibrosis Modeling after Acute Myocardial}

Infarction in Rabbits. A myocardial infarction model was successfully established by ligating the anterior descending 

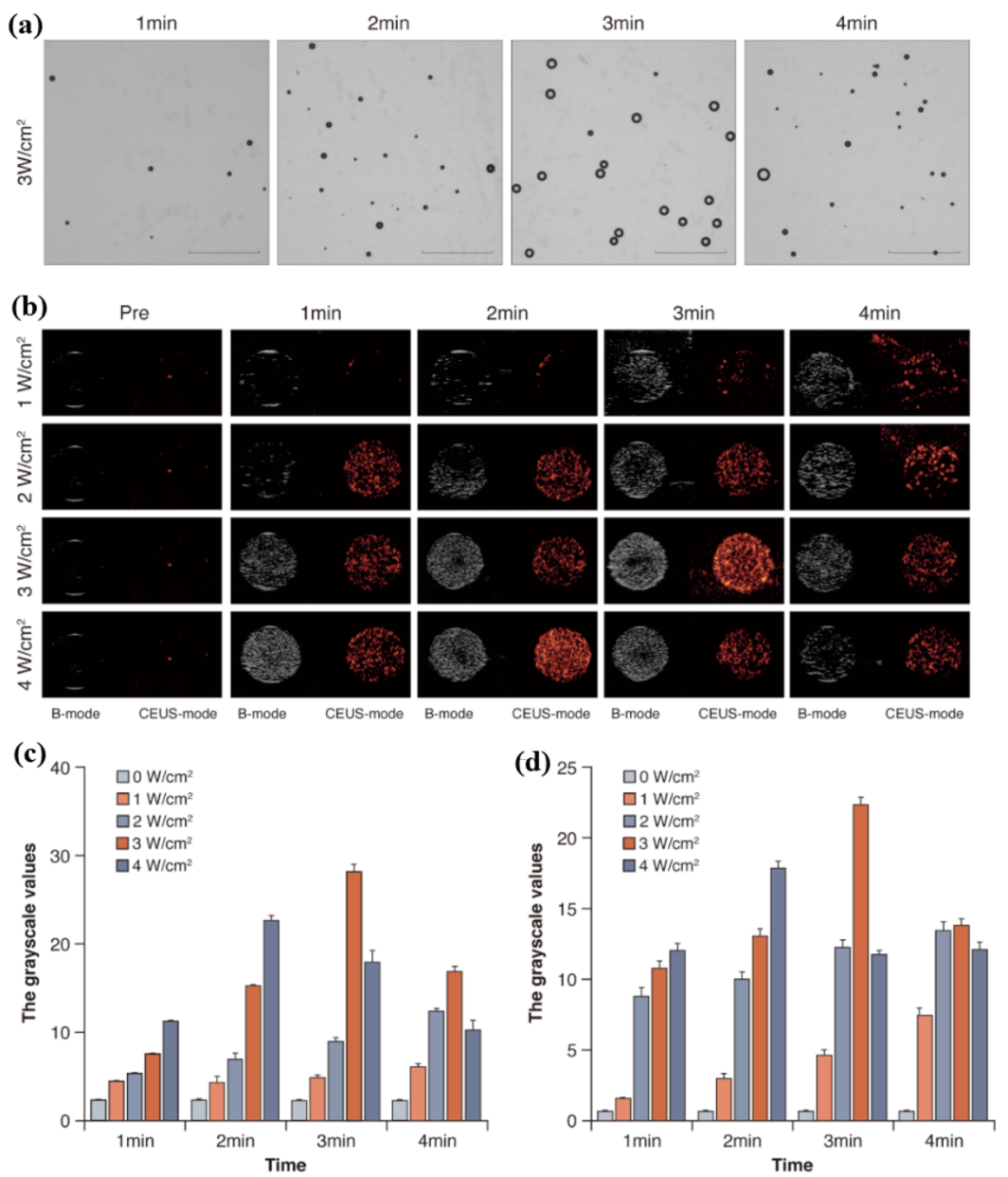

Figure 5. LIFU-triggered in vitro phase transition and ultrasound imaging of CNA35-PFP NPs. (a) Optical microscope graphics of phasetransformation after LIFU-irradiated treatment of CNA35-PFP NPs at $3 \mathrm{~W}$ for $3 \mathrm{~min}(100 \times)$. (b) Ultrasound imaging of CNA35-PFP NPs after irradiation by LIFU under different conditions. (c) Gray-scale values of B-mode imaging of CNA35-PFP NPs after LIFU irradiation under different conditions. (d) Gray-scale values of CEUS-mode imaging of CNA35-PFP NPs after LIFU irradiation under different conditions as shown in the corresponding figure.

coronary artery. A postoperative electrocardiogram showed that the ST segment of the chest lead was significantly elevated (Figure 6). One month after the operation, the echocardiogram showed that the anterior wall myocardium became thinner and the amplitude of motion was weakened. A pathological examination indicated myocardial infarction with fibrosis (Figure 7).

3.4. Ex Vivo CNA35-FITC-Targeted Binding Ability, Imaging Effect, and Quantitative Analysis. In the ex vivo experiment, the frozen sections of the myocardial fibrosis model and normal rabbit myocardium were selected. Both two groups were stained with CNA35-FITC and DAPI. After repeated washing, they were observed under a fluorescence confocal microscope. Type I collagen labeled with CNA35FITC showed the green fluorescence under confocal laser scanning microscopy (CLSM) observation, whereas the nuclei labeled by DAPI showed the blue fluorescence (Figure 8a). The ex vivo green fluorescence signal of the fibrotic myocardium in the myocardial infarction group was significantly enhanced as observed by CLSM, whereas only a small amount of green fluorescence was observed in the healthy rabbit myocardium. The results were consistent with the results of Sirius red staining (Figure 7). Hematoxylin and eosin (HE) staining, Masson staining, and Sirius red staining were (a)

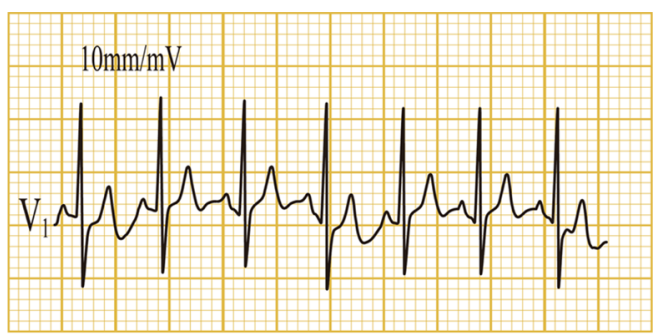

(b)

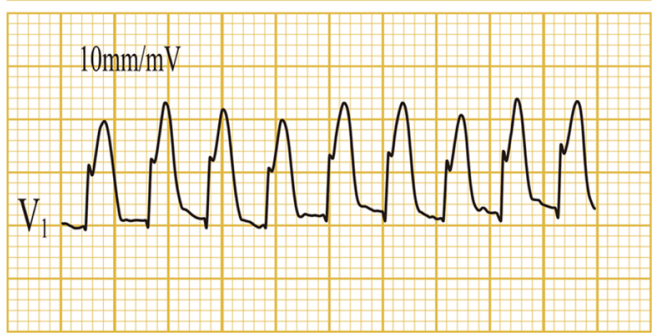

Figure 6. (a) Preoperative and (b) postoperative electrocardiogram of rabbit acute myocardial infarction modeling. The postoperative electrocardiogram showed that the $\mathrm{ST}$ segment of the $\mathrm{V}_{1}$ lead was successfully increased.

performed on the sections, respectively. Significant fibrosis was observed in the stained myocardial tissue (Figure 8a). The 

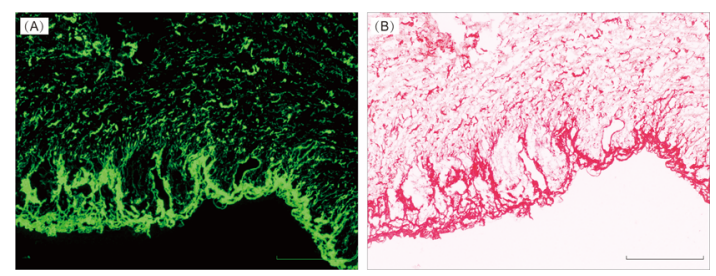

Figure 7. (A) Myocardial collagen fibers stained with CNA35-FITC and evaluated by fluorescence confocal microscopy showing green fluorescence. Their distribution was consistent with (B) the results of Sirius red staining.

results of the corresponding semiquantitative analysis of each group were in accordance with CLSM characterization (Table S2, Figure 8b).In this ex vivo experiment, we confirmed that CNA35 could well accumulate at the site of myocardial fibrosis. Comparatively, there was no apparent accumulation in the nonfibrotic region, which provides a basis for the subsequent in vivo experiments.

3.5. Cytotoxicity and in Vivo Safety Evaluation of CNA35-PFP NPs. The cultured CFs were identified by positive staining for vimentin and negative staining for CD31 and troponin I (Figure 9a-c). After incubation with CNA35PFP NPs for $24 \mathrm{~h}$, no significant cytotoxicity was observed in all concentrations of CNA35-PFP NPs (Figure 9d). Meanwhile, no damage in any of the organs was observed under a light microscope after $48 \mathrm{~h}$ of CNA35-PFP NP injection (Figure 10), indicating the relatively high biocompatibility of as-synthesized nanoprobes.

3.6. In Vivo Binding Assay of Type I Collagen FiberTargeted CNA35-FITC Nanoagents. For in vivo evaluation,
PFP NPs-DiI or CNA35-FITC-PFP NPs-DiI was injected through the ear vein in rabbits with myocardial infarction or normal controls (the control group and the myocardial infarction group received PFP NPs-DiI or CNA35-FITC-PFP NPs-DiI injection). Then, the heart was taken out in the dark to prepare frozen sections. Myocardial tissues were observed under a laser confocal microscope. The results showed that the green fluorescent collagen fibers and red fluorescent lipid phase-changeable nanoagents were observed in the myocardial fibrosis model group after the injection of CNA35-FITC-PFP NPs-DiI. However, in myocardial infarction rabbits injected with non-collagen targeted nanoparticles (PFP NPs-DiI) and normal rabbits injected with CNA35-FITC-PFP or NPs-DiI, neither green nor red fluorescence imaging was observed under laser confocal microscopy (Figure 11), indicating that CNA35PFP NPs are featured with an intriguing targeting capability to myocardial type I collagen with high stability in vivo. This result further demonstrates that the nanoagents as-prepared herein are able to cross the vascular endothelium to reach the fibrotic area.

3.7. In Vivo Ultrasound Molecular Imaging of Myocardial Fibrosis. For rabbits with myocardial infarction, the infarcted area was observed in the left ventricle anterior wall and/or anteroseptal wall by echocardiography. The thickness of the infarcted myocardium was thinner than that of the noninfarcted area $(0.093 \pm 0.012 \mathrm{~cm}$ vs $0.31 \pm 0.034$ $\mathrm{cm}, * * p<0.05)$, and the echo intensity seemed higher in the infarcted segments. As reported by Danila et al., ${ }^{35}$ strong blood pool enhancement was observed only $5 \mathrm{~min}$ after CNA35AuNP administration. According to Danila's report, we performed ultrasound observations $5 \mathrm{~min}$ after the injection
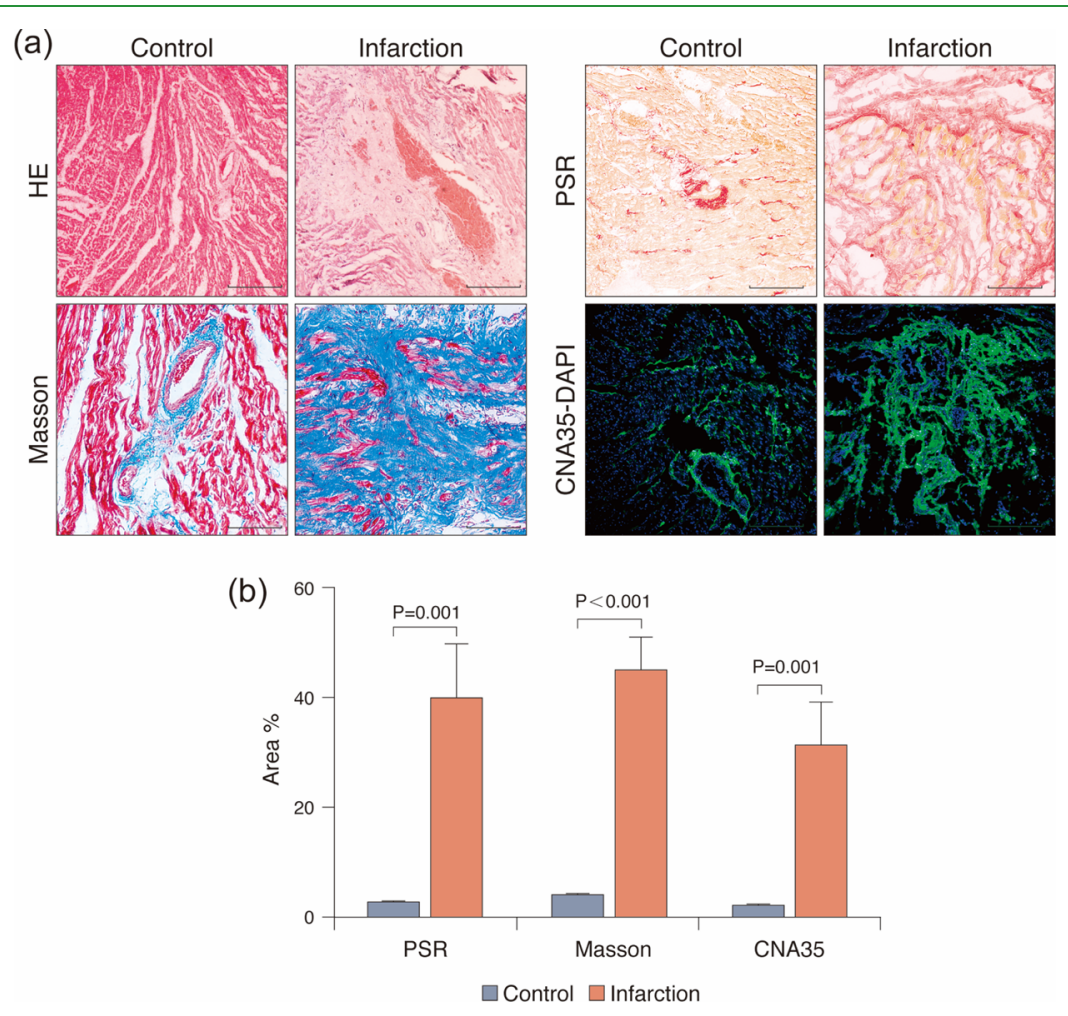

Figure 8. Staining results of myocardial fibrosis in myocardial infarction and normal group myocardial tissue, including (a) HE staining, Masson, Sirius red, and CNA35-FITC staining (scale bar: $500 \mu \mathrm{m}$ ). Significant collagen fibrosis was observed in the infarcted myocardium. (b) Semiquantitative analysis of the staining results of Masson staining, PSR staining, and CNA35-FITC in the control group and experimental group $(n=5), * * p<0.05$. 
(a)

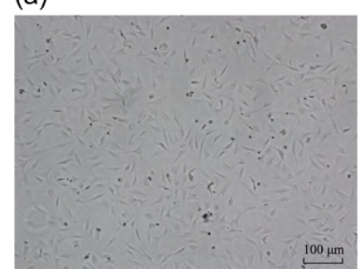

(b) DAPI

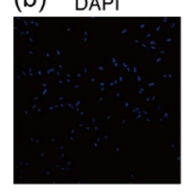

(c) DAPI
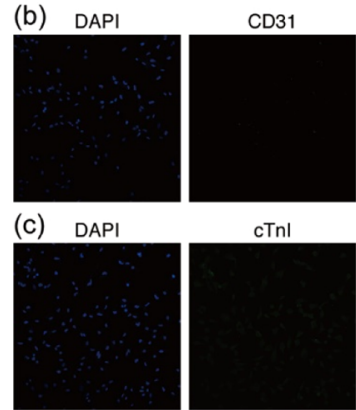

$c T n 1$

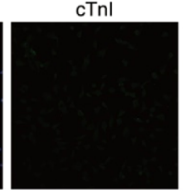

Vimentin
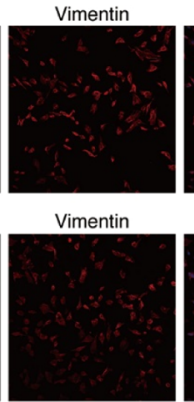

(d)

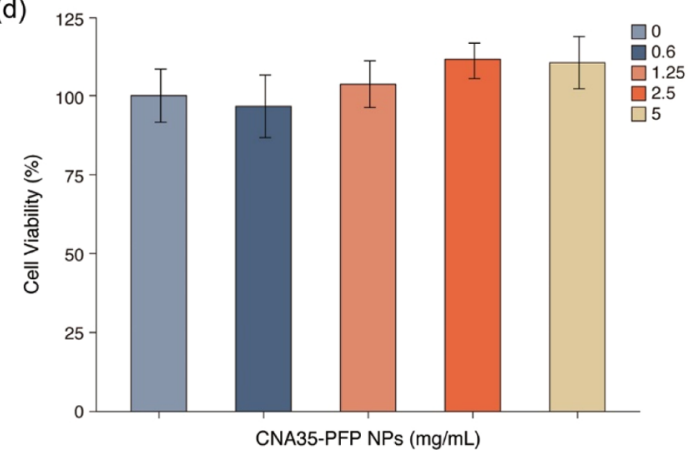

Figure 9. Ex vivo cytotoxicity study with cultured rat primary CFs. (a) Cultured CFs isolated from neonatal Sprague Dawley rats. $(b, c)$ CF identification with negative staining for $\mathrm{CD} 31$, troponin $\mathrm{I}$, and positive staining for vimentin. (d) Compared to control, no significant cytotoxicity was observed after being co-incubated with all concentrations of CNA35-PFP NPs for $24 \mathrm{~h}$.

of PFP NPs or CNA35-PFP NPs; no obvious enhancement was found in the infarction-related myocardium in both groups. After LIFU irradiation to the heart at the power of 3 $\mathrm{W} / \mathrm{cm}^{2}$ for $3 \mathrm{~min}$, also no contrast-enhanced effect was observed by echocardiography in rabbits that received noncollagen I-targeted nanoparticles (PFP NPs) (non-targeting group). However, in rabbits that received CNA35-labeled perfluoropentane nanoparticles (CNA35-PFP NPs) (targeting group), significant ultrasound signal enhancement in the infarcted area was observed 3 min after LIFU irradiation (Figure 12a), indicating that only collagen-targeted nanoparticles could reach and accumulate at the fibrotic myocardium. It also demonstrates that phase transition occurred in vivo after LIFU irradiation.

These experimental results confirmed that CNA35 NPs could cross the vascular endothelium, bind to myocardial fibrosis in a targeted manner, and enhance the ultrasoundimaging signal. Nanoparticles without CNA35 modification could not target the fibrotic myocardium, causing difficulty in inducing the contrast-enhanced effect for ultrasound imaging. In addition, no ultrasound enhancement was observed in the CNA35 NP group before LIFU irradiation, indicating that these nanoagents could hardly spontaneously produce a phase transition in vivo where LIFU triggering was necessary. This result was consistent with the results as observed in vitro.

The gray-scale values of each group at baseline, and before and after LIFU irradiation were statistically analyzed at Bmode and contrast mode. After LIFU irradiation, the gray-scale values in the CNA35-NP group were significantly higher than those before LIFU irradiation and at baseline $(* * p<0.05)$. Images at the same view were also observed in contrast mode, further eliminating interference from echoes in other parts (Figure 12b,c). The results of Sirius red staining were consistent with those obtained by ultrasonic imaging (Figure 12d).

This work validated the specific capability of CNA35-PFP NPs to diagnose myocardial fibrosis by ultrasound imaging, but this ability depends on the microcirculation at the infarction site. However, we performed the imaging study 30 days after myocardial infarction, which might provide enough time to form collateral circulation, and the nanoparticles could get to the fibrotic myocardium through the collateral pathways. In addition, we tested targeting of CNA35-PFP NPs in an animal model of myocardial infarction. It has not been revealed whether the target imaging effect could be observed in animal models with diffuse scarring. Future studies on different types of myocardial scarring in different animal models might refine these results. Furthermore, although no LIFU treatmentrelated death was observed in this work, the potential side effects such as arrhythmias and cardiac edema need to be further investigated.

\section{CONCLUSIONS}

In this work, we successfully constructed CNA35-labeled perfluoropentane nanoparticles (CNA35-PFP NPs) carrying a CNA35 molecular probe by the typical carbodiimide method.

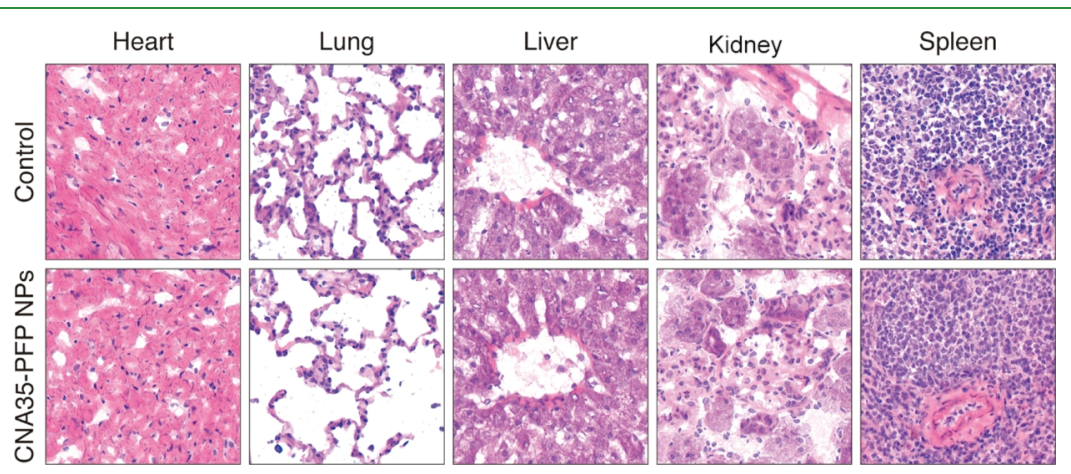

Figure 10. HE staining of the main organs including heart, lung, liver, kidney, and spleen under a light microscope 48 h after CNA35-PFP NP injection. No damage in any of the organs was observed (400×). 

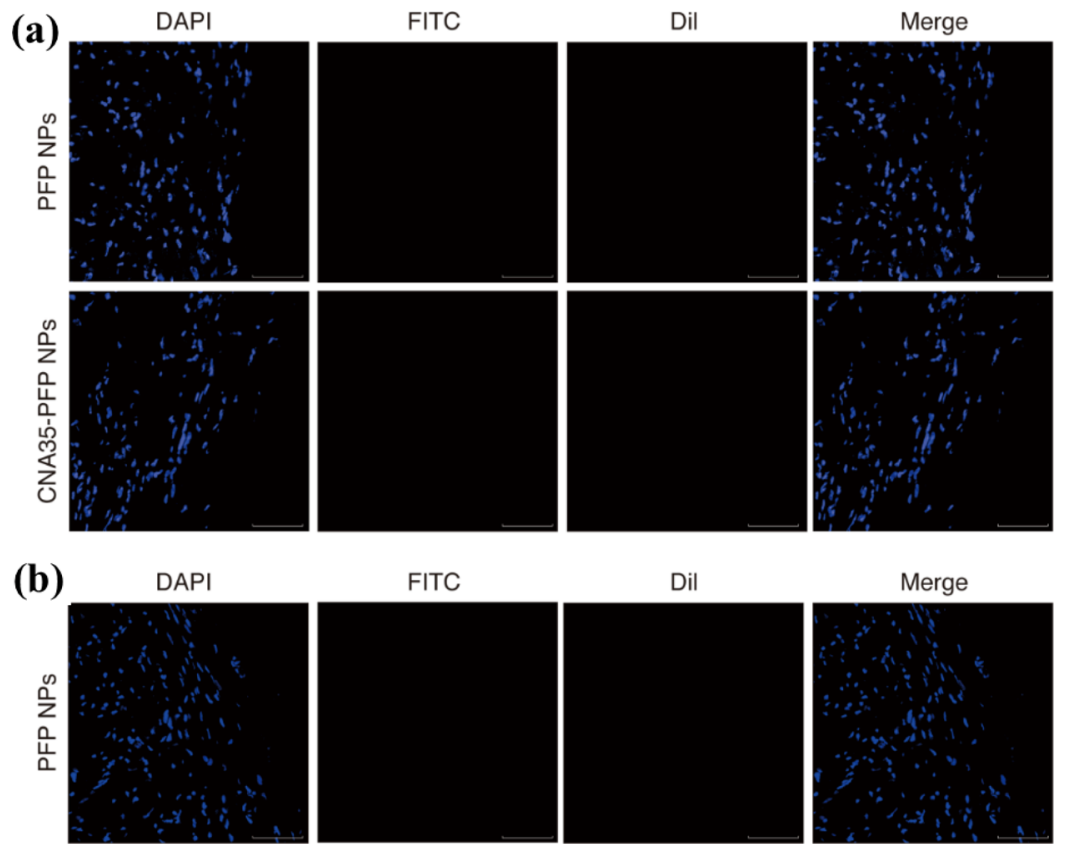

FITC
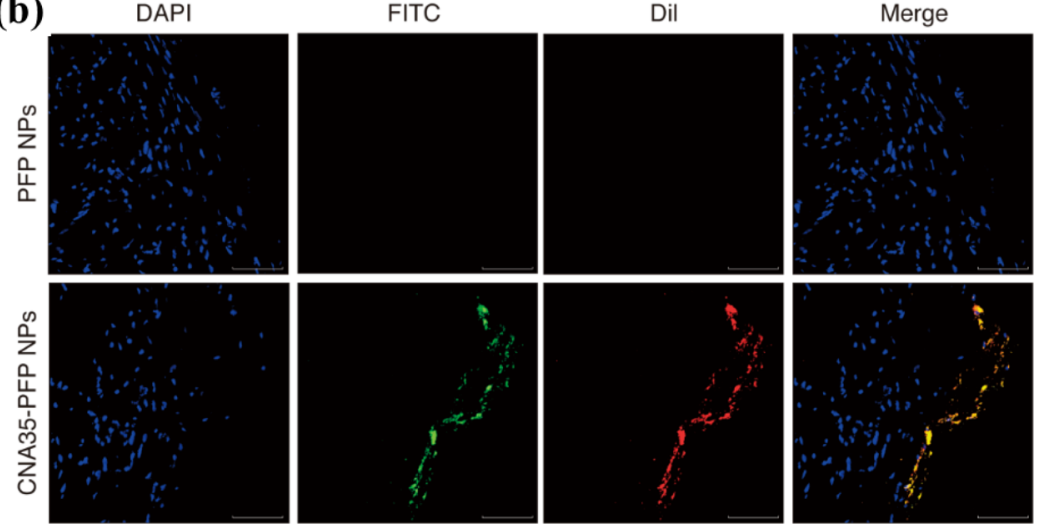

Figure 11. In vivo fluorescence imaging of PFP NPs and CNA35-PFP NPs. (a) The control group and (b) the infarct group were injected with PFP NPs-DiI and CNA35-FITC-PFP NPs-DiI. After $1 \mathrm{~h}$, the myocardial tissue was collected to prepare frozen sections and observed under a fluorescence confocal microscope. DAPI was used for nuclear localization. Green indicates fibrous collagen tissue, red indicates PFP NPs, and blue indicates the nucleus $(400 \times)$.

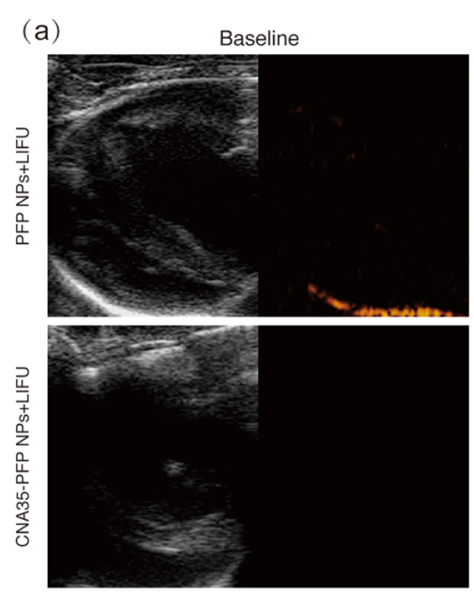

(b)

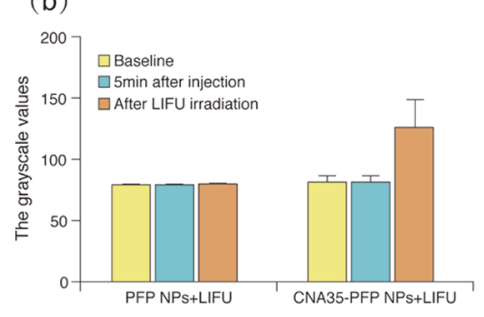

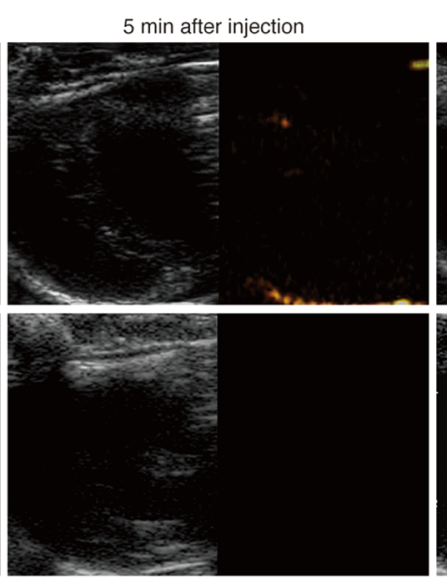

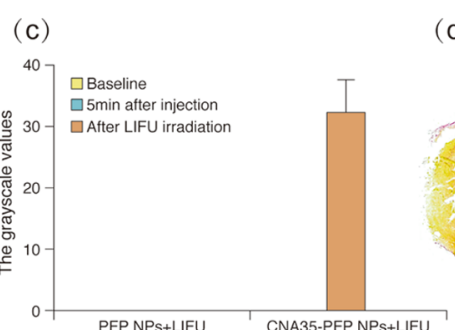

After LIFU irradiation

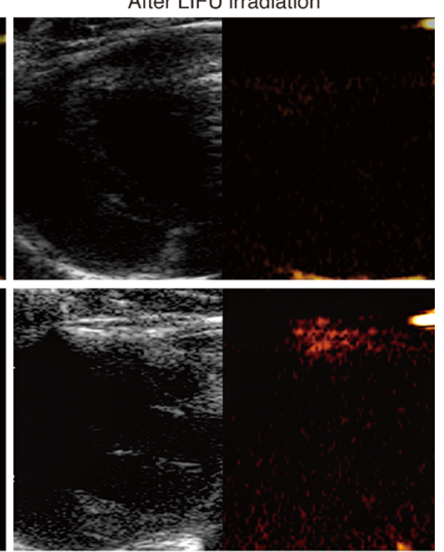

(d)

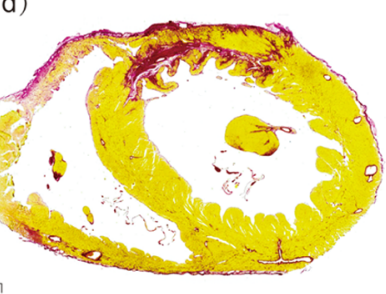

Figure 12. Ultrasound molecular imaging of CNA35 collagen-targeted fluorocarbon nanoparticles for myocardial fibrosis: (a) injection of PFP NPs and CNA35-PFP NPs, respectively, in B-mode (left) and contrast mode (right) before and after LIFU ultrasound focusing images, (b) changes in gray-scale values of each group in B-mode $(c)$, and CEUS mode (d). Collagen area of infarction group stained by PSR (0.6X). 
These targeted nanoagents conjugated with CNA35 are featured with attractive characteristics such as small nanoscale size, strong penetrability, and high specificity. On the basis of the ex vivo and in vivo evaluations, these phase-changeable nanoparticles conjugating with CNA35 could efficiently pass the vascular endothelium and specifically accumulate into the myocardial fibrous tissue. The fibrotic tissue can thus be visualized noninvasively using ultrasound imaging in combination with LIFU irradiation. This is the first time to realize the in situ ultrasound molecular imaging of myocardial fibrosis. On the basis of the results of this work, the precise targeted therapy for the myocardial cardiac might be facilitated by this intriguing ultrasound-imaging technique. Therefore, the asconstructed phase-changeable CNA35-PFP NPs in this work could potentially act as the highly promising molecular probe in the diagnosis and treatment of cardiac fibrosis.

\section{ASSOCIATED CONTENT}

\section{S Supporting Information}

The Supporting Information is available free of charge on the ACS Publications website at DOI: 10.1021/acsami.9b05999.

Hydrodynamic particle size distribution and zeta potential of CNA35-PFP NPs; the stability of CNA35PFP NPs on heating at $37.5{ }^{\circ} \mathrm{C}$; ultrasound imaging of CNA35-PFP NPs in different durations after LIFU irradiation; the distribution of fluorescence in major organs after CNA35-FITC-PFP NPs-DiI injection; the diameter and zeta potential of the two type of nanoparticles as measured by a dynamic light scattering analyzer; and semiquantitative analysis of the staining results of Masson staining, PSR staining, and CNA35FITC in the control group and experimental group (PDF)

\section{AUTHOR INFORMATION}

\section{Corresponding Author}

*E-mail: lingzy1977@163.com.

\section{ORCID}

\section{Zhiyu Ling: 0000-0003-1138-9528}

\section{Author Contributions}

Q.Z. and Y.Z. contributed equally to this paper, and should be considered as co-first authors. All the authors have approved the final version of the paper.

\section{Notes}

The authors declare no competing financial interest.

\section{ACKNOWLEDGMENTS}

We thank Dr. Yunlin Chen from the department of cardiology, the Second Affiliated Hospital of Chongqing Medical University for his work on the culture of rat primary CFs and in vitro cytotoxicity experiments. The authors gratefully acknowledge the financial support for this work from the Fund by National Natural Science Foundation of China (grant nos. 81571692, 81771847), outstanding young talents fund from the Second Affiliated Hospital of Chongqing Medical University.

\section{REFERENCES}

(1) Frangogiannis, N. G. Cardiac Fibrosis: Cell Biological Mechanisms, Molecular Pathways and Therapeutic Opportunities. Mol. Aspects Med. 2019, 65, 70-99.
(2) Burlew, B. S.; Weber, K. T. Connective Tissue and the Heart: Functional Significance and Regulatory Mechanisms. Cardiol. Clin. 2000, 18, 435-442.

(3) Burlew, B. S.; Weber, K. T. Cardiac Fibrosis as a Cause of Diastolic Dysfunction. Herz 2002, 27, 92-98.

(4) Gulati, A.; Jabbour, A.; Ismail, T. F.; Guha, K.; Khwaja, J.; Raza, S.; Morarji, K.; Brown, T. D. H.; Ismail, N. A.; Dweck, M. R.; Di Pietro, E.; Roughton, M.; Wage, R.; Daryani, Y.; O’Hanlon, R.; Sheppard, M. N.; Alpendurada, F.; Lyon, A. R.; Cook, S. A.; Cowie, M. R.; Assomull, R. G.; Pennell, D. J.; Prasad, S. K. Association of Fibrosis With Mortality and Sudden Cardiac Death in Patients with Nonischemic Dilated Cardiomyopathy. J. Am. Med. Assoc. 2013, 309, 896-908.

(5) Marra, M. P.; De Lazzari, M.; Zorzi, A.; Migliore, F.; Zilio, F.; Calore, C.; Vettor, G.; Tona, F.; Tarantini, G.; Cacciavillani, L. Impact of the Presence and Amount of Myocardial Fibrosis by Cardiac Magnetic Resonance on Arrhythmic Outcome and Sudden Cardiac Death in Nonischemic Dilated Cardiomyopathy. Heart Rhythm 2014, $11,856-863$.

(6) Gao, P.; Yee, R.; Gula, L.; Krahn, A. D.; Skanes, A.; Leong-Sit, P.; Klein, G. J.; Stirrat, J.; Fine, N.; Pallaveshi, L.; Wisenberg, G.; Thompson, T. R.; Prato, F.; Drangova, M.; White, J. A. Prediction of Arrhythmic Events in Ischemic and Dilated Cardiomyopathy Patients Referred for Implantable Cardiac Defibrillator: Evaluation of Multiple Scar Quantification Measures for Late Gadolinium Enhancement Magnetic Resonance Imaging. Circ. Cardiovasc. Imaging 2012, 5, 448456.

(7) Ordovas, K. G.; Higgins, C. B. Delayed Contrast Enhancement on MR Images of Myocardium: Past, Present, Future. Radiology 2011, $261,358-374$.

(8) Dewey, M.; Schink, T.; Dewey, C. F. Claustrophobia during Magnetic Resonance Imaging: Cohort Study in Over 55,000 Patients. J. Magn. Reson. Imaging 2007, 26, 1322-1327.

(9) Nakatsuka, M. A.; Barback, C. V.; Fitch, K. R.; Farwell, A. R.; Esener, S. C.; Mattrey, R. F.; Cha, J. N.; Goodwin, A. P. In Vivo Ultrasound Visualization of Non-Occlusive Blood Clots with Thrombin-Sensitive Contrast Agents. Biomaterials 2013, 34, 95599565.

(10) Wen, Q.; Wan, S.; Liu, Z.; Xu, S.; Wang, H.; Yang, B. Ultrasound Contrast Agents and Ultrasound Molecular Imaging. J. Nanosci. Nanotechnol. 2014, 14, 190-209.

(11) Lanza, G. M.; Yu, X.; Winter, P. M.; Abendschein, D. R.; Karukstis, K. K.; Scott, M. J.; Chinen, L. K.; Fuhrhop, R. W.; Scherrer, D. E.; Wickline, S. A. Targeted Antiproliferative Drug Delivery to Vascular Smooth Muscle Cells with a Magnetic Resonance Imaging Nanoparticle Contrast Agent: Implications for Rational Therapy of Restenosis. Circulation 2002, 106, 2842-2847.

(12) Winter, P. M.; Morawski, A. M.; Caruthers, S. D.; Fuhrhop, R. W.; Zhang, H.; Williams, T. A.; Allen, J. S.; Lacy, E. K.; Robertson, J. D.; Lanza, G. M.; Wickline, S. A. Molecular Imaging of Angiogenesis in Early-Stage Atherosclerosis with AVB3-Integrin-Targeted Nanoparticles. Circulation 2003, 108, 2270-2274.

(13) Zhao, H.; Wu, M.; Zhu, L.; Tian, Y.; Wu, M.; Li, Y.; Deng, L.; Jiang, W.; Shen, W.; Wang, Z.; Mei, Z.; Li, P.; Ran, H.; Zhou, Z.; Ren, J. Cell-Penetrating Peptide-Modified Targeted Drug-Loaded PhaseTransformation Lipid Nanoparticles Combined with Low-Intensity Focused Ultrasound For Precision Theranostics Against Hepatocellular Carcinoma. Theranostics 2018, 8, 1892-1910.

(14) Gao, Y.; Chen, S.; Li, W.; Wang, H.; Xiao, K.; Wu, L.; Li, Y.; Li, H.; Li, H.; Zhu, Y. An Experimental Study of Ovarian Cancer Imaging and Therapy by Paclitaxel-Loaded Phase-Transformation Lipid Nanoparticles Combined with Low-Intensity Focused Ultrasound. Biochem. Biophys. Res. Commun. 2018, 504, 203-210.

(15) Li, M.; Luo, H.; Zhang, W.; He, K.; Chen, Y.; Liu, J.; Chen, J.; Wang, D.; Hao, L.; Ran, H.; Zheng, Y.; Wang, Z.; Li, P. Phase-Shift, Targeted Nanoparticles for Ultrasound Molecular Imaging by Low Intensity Focused Ultrasound Irradiation. Int. J. Nanomed. 2018, 13, $3907-3920$ 
(16) Liu, J.; Shang, T.; Wang, F.; Cao, Y.; Hao, L.; Ren, J.; Ran, H.; Wang, Z.; Li, P.; Du, Z. Low-Intensity Focused Ultrasound (LIFU)Induced Acoustic Droplet Vaporization in Phase-Transition Perfluoropentane Nanodroplets Modified By Folate for Ultrasound Molecular Imaging. Int. J. Nanomed. 2017, 12, 911-923.

(17) Perera, R. H.; Hernandez, C.; Zhou, H.; Kota, P.; Burke, A.; Exner, A. A. Ultrasound Imaging Beyond the Vasculature with New Generation Contrast Agents. Wiley Interdiscip. Rev.: Nanomed. Nanobiotechnol. 2015, 7, 593-608.

(18) Leuschner, F.; Nahrendorf, M. Molecular Imaging of Coronary Atherosclerosis and Myocardial Infarction. Circ. Res. 2011, 108, 593606.

(19) Pysz, M. A.; Foygel, K.; Rosenberg, J.; Gambhir, S. S.; Schneider, M.; Willmann, J. K. Antiangiogenic Cancer Therapy: Monitoring with Molecular US and a Clinically Translatable Contrast Agent (BR55). Radiology 2010, 256, 519-527.

(20) Zhang, P.; Cao, Y.; Chen, H.; Zhou, B.; Hu, W.; Zhang, L. Preparation And Evaluation of Glycyrrhetinic Acid-Modified And Honokiol-Loaded Acoustic Nanodroplets for Targeted Tumor Imaging and Therapy with Low-Boiling-Point Phase-Change Perfluorocarbon. J. Mater. Chem. B 2017, 5, 5845-5853.

(21) Tang, H.; Guo, Y.; Peng, L.; Fang, H.; Wang, Z.; Zheng, Y.; Ran, H.; Chen, Y. In Vivo Targeted, Responsive and Synergistic Cancer Nanotheranostics by MRI-Guided Synergistic HIFU Ablation and Chemotherapy. ACS Appl. Mater. Interfaces 2018, 10, 1542815441.

(22) You, Y.; Wang, Z.; Ran, H.; Zheng, Y.; Wang, D.; Xu, J.; Wang, Z.; Chen, Y.; Li, P. Nanoparticle-Enhanced Synergistic HIFU Ablation and Transarterial Chemoembolization for Efficient Cancer Therapy. Nanoscale 2016, 8, 4324-4339.

(23) Sun, J.; Yin, M.; Zhu, S.; Liu, L.; Zhu, Y.; Wang, Z.; Xu, R. X.; Chang, S. Ultrasound-Mediated Destruction of Oxygen and Paclitaxel Loaded Lipid Microbubbles for Combination Therapy in Hypoxic Ovarian Cancer Cells. Ultrason. Sonochem. 2016, 28, 319-326.

(24) Deng, L.; Cai, X.; Sheng, D.; Yang, Y.; Strohm, E. M.; Wang, Z.; Ran, H.; Wang, D.; Zheng, Y.; Li, P.; Shang, T.; Ling, Y.; Wang, F.; Sun, Y. A Laser-Activated Biocompatible Theranostic Nanoagent for Targeted Multimodal Imaging and Photothermal Therapy. Theranostics 2017, 7, 4410-4423.

(25) Zong, Y.; Xu, Y.; Liang, X.; Keene, D. R.; Höök, A.; Gurusiddappa, S.; Höök, M.; Narayana, S. V. L. A "Collagen Hug" Model for Staphylococcus aureus CNA binding to collagen. EMBO J. 2005, 24, 4224-4236.

(26) Krahn, K. N.; Bouten, C. V. C.; van Tuijl, S.; van Zandvoort, M. A. M. J.; Merkx, M. Fluorescently Labeled Collagen Binding Proteins Allow Specific Visualization of Collagen in Tissues and Live Cell Culture. Anal. Biochem. 2006, 350, 177-185.

(27) Weber, K. T.; Janicki, J. S.; Shroff, S. G.; Pick, R.; Chen, R. M.; Bashey, R. I. Collagen Remodeling of The Pressure-Overloaded, Hypertrophied Nonhuman Primate Myocardium. Circ. Res. 1988, 62, $757-765$.

(28) Horn, M. A.; Trafford, A. W. Aging and the Cardiac Collagen Matrix: Novel Mediators of Fibrotic Remodelling. J. Mol. Cell. Cardiol. 2016, 93, 175-185.

(29) Talman, V.; Ruskoaho, H. Cardiac Fibrosis in Myocardial Infarction-from Repair and Remodeling to Regeneration. Cell Tissue Res. 2016, 365, 563-581.

(30) Goette, A.; Kalman, J. M.; Aguinaga, L.; Akar, J.; Cabrera, J. A.; Chen, S. A.; Chugh, S. S.; Corradi, D.; D’Avila, A.; Dobrev, D.; Fenelon, G.; Gonzalez, M.; Hatem, S. N.; Helm, R.; Hindricks, G.; Ho, S. Y.; Hoit, B.; Jalife, J.; Kim, Y.-H.; Lip, G. Y. H.; Ma, C.-S.; Marcus, G. M.; Murray, K.; Nogami, A.; Sanders, P.; Uribe, W.; Van Wagoner, D. R.; Nattel, S. EHRA/HRS/APHRS/SOLAECE Expert Consensus on Atrial Cardiomyopathies: Definition, Characterization, and Clinical Implication. Heart Rhythm 2017, 14, e3-e40.

(31) Chen, J.; Lee, S. K.; Abd-Elgaliel, W. R.; Liang, L.; Galende, E.Y.; Hajjar, R. J.; Tung, C.-H. Assessment of Cardiovascular Fibrosis using Novel Fluorescent Probes. PLoS One 2011, 6, e19097.
(32) van Bochove, G. S.; Sanders, H. M. H. F.; de Smet, M.; Keizer, H. M.; Mulder, W. J. M.; Krams, R.; Strijkers, G. J.; Nicolay, K. Molecular MR Imaging of Collagen in Mouse Atherosclerosis by Using Paramagnetic CNA35 Micelles. Eur. J. Inorg. Chem. 2012, 2012, $2115-2125$

(33) Kee, P. H.; Danila, D. CT Imaging of Myocardial Scar Burden with CNA35-Conjugated Gold Nanoparticles. Nanomedicine 2018, 14, 1941-1947.

(34) Tao, Q; Piers, S. R. D.; Lamb, H. J.; van der Geest, R. J. Automated Left Ventricle Segmentation in Late GadoliniumEnhanced MRI for Objective Myocardial Scar Assessment. J. Magn. Reson. Imaging 2015, 42, 390-399.

(35) Danila, D.; Johnson, E.; Kee, P. CT Imaging of Myocardial Scars with Collagen-Targeting Gold Nanoparticles. Nanomedicine 2013, 9, 1067-1076.

(36) Mees, G.; Dierckx, R.; Mertens, K.; Vermeire, S.; Van Steenkiste, M.; Reutelingsperger, C.; D’Asseler, Y.; Peremans, K.; Van Damme, N.; Van de Wiele, C. ${ }^{99} \mathrm{mTc}$-labeled Tricarbonyl HisCNA35 as an Imaging Agent for the Detection of Tumor Vasculature. J. Nucl. Med. 2012, 53, 464-471.

(37) Chen, Y.-1.; Fan, J.; Cao, L.; Han, T.-1.; Zeng, M.; Xu, Y.; Ling, Z.; Yin, Y. Unique Mechanistic Insights into the Beneficial Effects of Angiotensin-(1-7) on the Prevention Of Cardiac Fibrosis: A Metabolomic Analysis of Primary Cardiac Fibroblasts. Exp. Cell Res. 2019, 378, 158-170.

(38) Ren, J. L.; Xu, C. S.; Zhou, Z. Y.; Zhang, Y.; Li, X. S.; Zheng, Y. Y.; Ran, H. T.; Wang, Z. G. A Novel Ultrasound Microbubble Carrying Gene and Tat Peptide: Preparation and Characterization. Acad. Radiol. 2009, 16, 1457-1465.

(39) Kong, P.; Christia, P.; Frangogiannis, N. G. The Pathogenesis of Cardiac Fibrosis. Cell. Mol. Life Sci. 2014, 71, 549-574.

(40) Li, L.; Zhao, Q.; Kong, W. Extracellular Matrix Remodeling and Cardiac Fibrosis. Matrix Biol. 2018, 68-69, 490-506.

(41) Boerboom, R. A.; Krahn, K. N.; Megens, R. T. A.; van Zandvoort, M. A. M. J.; Merkx, M.; Bouten, C. V. C. High Resolution Imaging of Collagen Organisation and Synthesis Using a Versatile Collagen Specific Probe. J. Struct. Biol. 2007, 159, 392-399.

(42) de Jong, S.; van Middendorp, L. B.; Hermans, R. H.; de Bakker, J. M.; Bierhuizen, M. F.; Prinzen, F. W.; van Rijen, H. V.; Losen, M.; Vos, M. A.; van Zandvoort, M. A. Ex Vivo and in Vivo Administration of Fluorescent CNA35 Specifically Marks Cardiac Fibrosis. Mol. Imaging 2014, 13, 1-9.

(43) Unnikrishnan, S.; Klibanov, A. L. Microbubbles as Ultrasound Contrast Agents for Molecular Imaging: Preparation and Application. AJR Am. J. Roentgenol. 2012, 199, 292-299.

(44) Garg, P.; Saunders, L. C.; Swift, A. J.; Wild, J. M.; Plein, S. Role of Cardiac T1 Mapping and Extracellular Volume in The Assessment of Myocardial Infarction. Anatol. J. Cardiol. 2018, 19, 404-411.

(45) Schutt, E. G.; Klein, D. H.; Mattrey, R. M.; Riess, J. G. Injectable Microbubbles as Contrast Agents for Diagnostic Ultrasound Imaging: The Key Role of Perfluorochemicals. Angew. Chem., Int. Ed. Engl. 2003, 42, 3218-3235.

(46) Flaim, S. F. Pharmacokinetics and Side Effects of Perfluorocarbon-Based Blood Substitutes. Artif. Cells Blood Substit. Immobil. Biotechno. 1994, 22, 1043-1054.

(47) Mattrey, R. F. The Potential Role of Perfluorochemicals (Pfcs) in Diagnostic Imaging. Artif. Cells, Blood Substitutes, Biotechnol. 1994, 22, 295-313.

(48) Sheng, D.; Liu, T.; Deng, L.; Zhang, L.; Li, X.; Xu, J.; Hao, L.; Li, P.; Ran, H.; Chen, H.; Wang, Z. Perfluorooctyl Bromide \& Indocyanine Green Co-Loaded Nanoliposomes for Enhanced Multimodal Imaging-Guided Phototherapy. Biomaterials 2018, 165, 1-13.

(49) Rapoport, N. Y.; Efros, A. L.; Christensen, D. A.; Kennedy, A. M.; Nam, K.-H. Microbubble Generation in Phase-Shift Nanoemulsions used as Anticancer Drug Carriers. Bubble Sci. Eng. Technol. 2009, 1, 31-39.

(50) Rapoport, N.; Christensen, D. A.; Kennedy, A. M.; Nam, K.-H. Cavitation Properties of Block Copolymer Stabilized Phase-Shift 
Nanoemulsions used as Drug Carriers. Ultrasound Med. Biol. 2010, 36,

419-429.

(51) Zhu, L.; Zhao, H.; Zhou, Z.; Xia, Y.; Wang, Z.; Ran, H.; Li, P.; Ren, J. Peptide-Functionalized Phase-Transformation Nanoparticles for Low Intensity Focused Ultrasound-Assisted Tumor Imaging and Therapy. Nano Lett. 2018, 18, 1831-1841. 